\title{
A Dependability Management Mechanism for Ubiquitous Computing Systems*
}

\author{
Changyeol Choi and Sungsoo Kim \\ Graduate School of Information and Communication, Ajou University, Suwon, Korea \\ \{clchoi, sskim\}@ajou.ac.kr
}

\begin{abstract}
Dependability for a ubiquitous computing system must be guaranteed for each single component of a system and for the whole system, whichdesigned to fulfill a certain task-might be more than just a sum of its components. Ubiquitous computing systems must enable the testing not only each of software components separately but also of the whole system. In this paper, we propose a management mechanism for applying software rejuvenation technology into a ubiquitous computing system. It adopts the automatic monitoring scheme, the automatic analysis scheme, the autonomic plan and the execution scheme to suggest the optimal configuration alternative of a ubiquitous computing system. We validate the autonomic fault management scheme based on a workload model derived from the system log analysis.
\end{abstract}

\section{Introduction}

Computing has spread from a desktop computer to several areas such as automobiles, gadgets, telecommunications and the Internet; that is, hundreds of internet-enabled computers per human being, none of them resembling a conventional keyboard-andmonitor machine. Particularly, a ubiquitous computing system could be constructed not only with one computer but with networks of computers (or other devices with computing power) embedded in everyday objects where computers are made available throughout the physical environment [1] and must be able to operate for 24 hours/7 days with the minimum human intervention. For this, dependability must be guaranteed for each single component of a system and for the whole system, whichdesigned to fulfill a certain task-might be more than just a sum of its components. In order to deal with faults efficiently, modular and structured systems-enable loosely coupled, componentized systems, such as Sun J2EE[2] and Micro soft .NET[3]-are mostly preferable. However, when a certain number of components fail owing to unpredicted failures, the system will fail to guarantee service quality to a large number of users. In addition to hardware failures, many unexpected system faults are caused by software failures $[4,5,6]$.

Software-aging phenomenon, a dormant form of software faults, can lead to data loss, communication disruption, and can be aggravated by malfunction such as memory leak, buffer overflow, data corruption, or numerical error accumulation, etc.

This research is supported by the Ubiquitous Autonomic Computing and Network Project, the Ministry of Information and Communication (MIC) 21st Century Frontier R\&D Program in Korea. 
Software rejuvenation is based on the idea of preventive maintenance applied to the software context that is widely employed in different engineering disciplines $[7,8,9,10]$. Software rejuvenation is a proactive fault management technique aimed at cleaning up the system internal state to prevent the occurrence of more severe crash failures in the future. It involves occasionally terminating an application or a system, cleaning its internal state and restarting it. Garbage collection, flushing operating system kernel tables, and reinitializing internal data structures are some examples of what cleaning the internal state of software might involve. Recent studies of the software rejuvenation technique stress the importance of the analysis on availability, downtime cost, and other methodology to improve system availability. However, it is necessary to address the management complexity issues by using technology to manage the software rejuvenation technology with the minimum human intervention. Moreover, they must enable the testing not only each of software components separately but also of the whole system. So, in this paper, we propose a management mechanism for applying software rejuvenation technology into a ubiquitous computing system. It adopts the automatic monitoring scheme to collect, aggregate, filter, and track related metrics, the automatic analysis scheme to extend our previous work[10], the autonomic plan and execution scheme to suggest the optimal configuration alternative of a ubiquitous computing system. We validate the autonomic fault management scheme based on a workload model derived from the system log analysis. The rest of this paper is organized as follows. The autonomic fault management mechanisms are presented in section 2. Section 3 describes how to get the metrics, analyze the availability, and make the suggestion for the dependability requirements in detail. Section 4 presents the analytic and simulation results based on experiments on different system parameters. Section 5 concludes the paper.

\section{Autonomic Fault Management Mechanisms}

The CIM(common information model) standards promoted by DMTF (distributed management task force) are a way to systematize the available information about the computing environment in which semantically rich management information between systems could be exchanged [11]. The approach uses a uniform modeling formalism that, together with the basic scenario of object-oriented constructs, supports the cooperative development of an object-oriented schema across multiple organizations. So, we describe with CIM and UML (Unified Modeling Language) the autonomic fault managements for a ubiquitous computing system-divided into two parts: the dependability manger for the plan and execution and the observation manager for the monitor and analysis (refer in Figure 1).

A class represents a concept within the system being modeled are denoted by the rectangular boxes in the diagram of Fig. 1. Classes contain the class name, the point data structure that has been defined for the class, and the behavior that can be performed on instances of this class. Multiplicity represents the number of instances of each class that form part of the relation with the lines connecting the classes, for examples, "a Dependability Manager controls one or many Observation Manager; a Observation Manager is managed by one Dependability Manager." A brief description of the classes in alphabetical order follows for the dependability manager and the observation manager, respectively. 


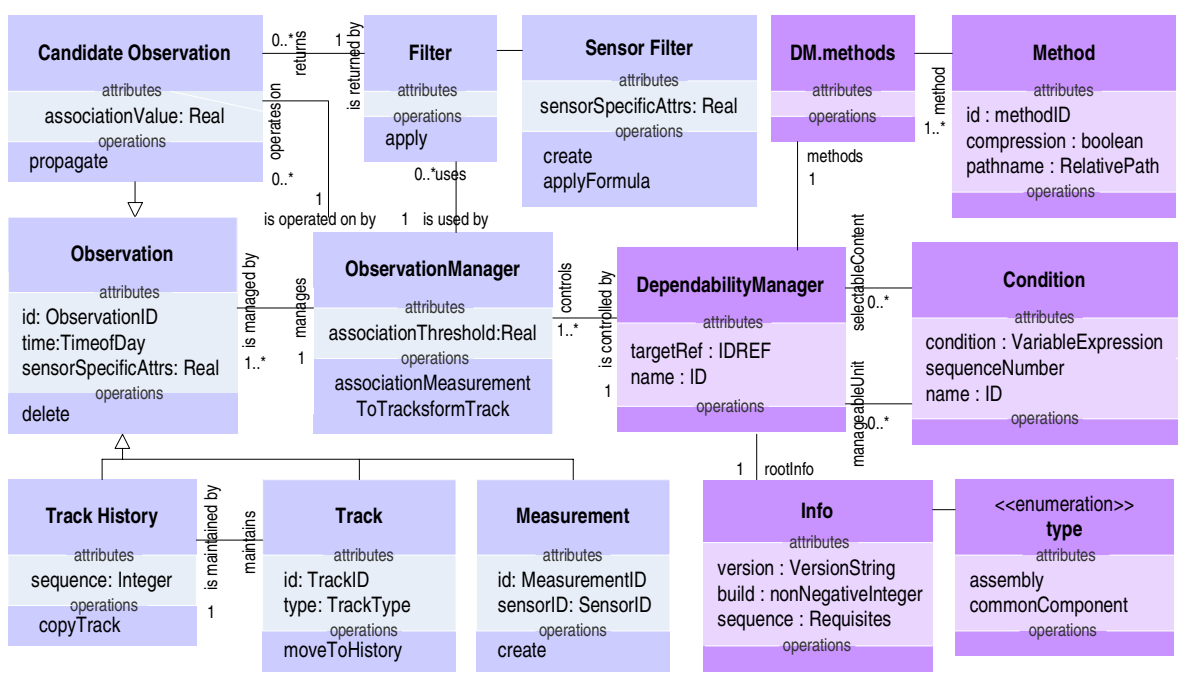

Fig. 1. UML representation of autonomic fault management mechanisms

\subsection{Dependability Manager}

Dependability Manager (DM) plays a core role in the autonomic fault manage-ments i $\mathrm{n}$ order to apply software rejuvenation technology into a ubiquitous computing system . It interprets the quality of applications and systems against a predefined service level , and finally invokes methods to correct a problem if necessary. In order to make a pla $\mathrm{n}$ and take a countermeasure, DM cooperates with the observation manager that analy zes the properties of objects aggregated in the model and generates indications about $t$ he status of those resources.

- Condition: the generic type describing any contained component defined within a u biquitous computing system. The condition is a boolean variable expression. When $t$ he condition is false, the DM invokes any method.

- Method: a composite of action definitions including a method identifier. Some of th e actions defined in a method may specify the execution of custom code with the pa th in order to satisfy some given requirements.

- Info: the definition for describing the characteristics of the system information installed on the version and the requisite target to satisfy a software requirement.

\subsection{Observation Manager}

The observation manager adopts the automatic monitoring and analysis scheme to coll ect, aggregate, filter, track related metrics, and finally generate indications about the st atus of a ubiquitous computing system.

- Filter: base class for all sensor filters. Application of the filter will then identify the tracks or measurements that lie within the volume determined by the filter.

- Measurement: all measurements provided by a sensor or communications interface and received by the Observation Manager. The attributes in this class have not been fully defined. 
- Observation: object data managed within the fault management domain. Candidate observation is created for each track or measurement that matches the filter criteria.

- Track: State data about a track object. The attributes in this class have not been fully defined and record the history associated to a specific track.

\section{Automation Scheme}

We divide the hazards of a ubiquitous computing system into three errors: environment and hardware structure error, system error, human error (refer in Figure 2). The system comes to an emergency stop if it fails to detect, prevent, or recover at least one of the three errors. Because many unexpected system faults are caused by software failures, we concentrate on system error in operation-sensor error, controller error, and actuator error. For automatic monitor, the reference model for a resource, component, or system must be constructed. The observation reference model controls the data gathering and interprets the data according to best-practices algorithms in order to detect the unpredicted fault phenomenon condition. For an example, we build up the observation reference model for a memory leakage phenomenon.

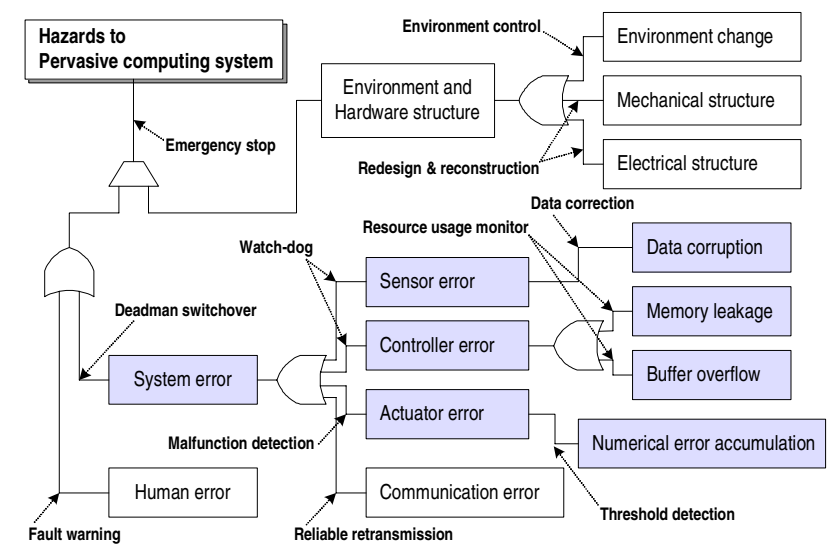

Fig. 2. Fault classification and possible events

A memory leakage occurs, when it is short of available memory with high working set and deficient in memory for private bytes of a specific process used in a software component. This phenomenon can be detected by tracking the state change of objects such as memory and process from the past state[12]. That is, the number of bytes used for the working set of the process, the number of bytes committed to a process, the total number of page faults caused by a process or the total memory available might be examined. If the portion of total working set in memory exceeds one of available memory, the shortage of memory with high working set occurs. If the current number of process private bytes exceeds the previous value of them accidentally, then the deficiency of private memory is observed, too. However, only quantitative monitoring and analysis is incomplete for rigorous fault management. It is necessary to adopt best- 
practices fault injection algorithm[13,14] for obtaining the failure rate of a software object (application, component, service, or interface) because the software aging phenomenon would occur in long mission time. Thus, the dependability manager has the capability to evaluate the robustness of its software object for itself. In addition to the functioning software to perform system checking, loader, applications, etc. an important design issue for the ubiquitous computing system is related to the number of components that should be placed in different operational modes: operations, repair, dormant, etc. The serviceable number of components may decline because of software aging phenomenon after a long mission and then, the number of components in dormant mode must be incremented to that degree. A component having dormant software failure can switch to operation mode by software rejuvenation or will come down to system failure. In the case of failure, that component must be repaired. A fault-repair model is necessary to carry out the analysis.
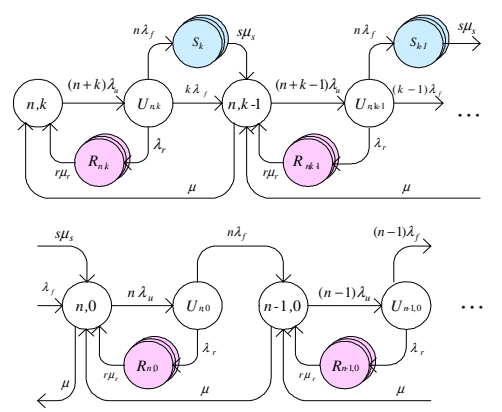

(a) all state
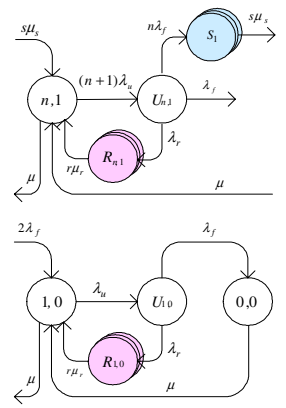

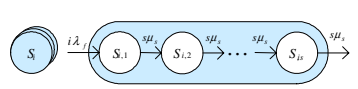

(b) rejuvenation state

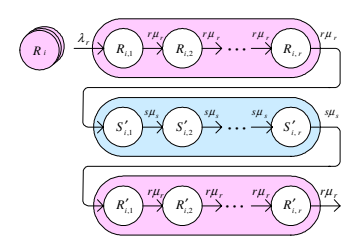

(c) switchover state

Fig. 3. State transition diagram for availability analysis of a ubiquitous computing system

The state transition diagram of the ubiquitous computing system that takes into account software rejuvenation and switchover is presented in Fig. 3. The two-tuple $\{(n, k),(n, k-1), \ldots,(1,0)\}$ annotated in each circle represents the number of primary and backup components in normal states respectively. After a certain period of mission time, the system may transit from one of the normal states to one of the unstable states $\left\{U_{n, k}, U_{n, k-1}, \ldots, U_{n, 0}, \ldots, U_{1,0}\right\}$ with rate $i \cdot \lambda_{u}$ where $i$ is the number of components, $i=n+k$ ( $n$ : the number of active components, $k$ : the number of backup components, $\lambda_{u}$ : the unstable rate of altering from healthy to faint). If a component is in an unstable state, its state can change to 1) the rejuvenation state, at rate $\lambda_{r}$ of operating the rejuvenation process, so that all components become free from faint conditions due to software aging, 2) the switchover state, at rate $n \cdot \lambda_{f}$, so that one backup component takes over the task of a faulty primary component when a primary component fails, or 3 ) the shut-down state at the rate $m \cdot \lambda_{f}$ where a backup component is shut down ( $m$ : the number of backup components), when a backup component fails. After rejuvenation, the system state departs from one of the unstable states. In rejuvenation states $\left\{R_{n, k}, R_{n, k-1}, \ldots, R_{1,0}\right\}$, not only one half of primary components, $\lceil n / 2\rceil$, but also one half of backup components, $\lceil k / 2\rceil$, are rejuvenated and followed by 
the other half. During transitions of the software rejuvenation, it is necessary for primary and backup components to subsume for each other of their computing tasks so that computing service would not be stopped at any time. Thus, one half of primary components waiting for the rejuvenation must keep service and one half of backup components waiting for the rejuvenation take over services of one half of primary components being rejuvenated. The state $(0,0)$ denotes that all components fail.

$$
P_{n+k}=\left[\begin{array}{l}
1+\sum_{i=0}^{n+k-1}\left\{\left(\frac{\lambda_{f} \cdot \lambda_{u}}{\mu}\right)^{n+k-i} \cdot\left(\prod_{l=0}^{n+k-1-i} \frac{(n+k-l)^{2}}{\lambda_{r}+(n+k-l) \cdot \lambda_{f}}\right) \cdot\left(1+\frac{\mu}{(i+1) \cdot \lambda_{f}} \cdot\left(1+\frac{2 \cdot \lambda_{r}}{r \cdot \mu_{r}}+\frac{\lambda_{r}}{s \cdot \mu_{s}}\right)\right)\right\} \\
+\sum_{i=0}^{k}\left\{\left(\frac{\lambda_{f} \cdot \lambda_{u}}{\mu}\right)^{k-i} \cdot\left(\prod_{l=0}^{k-i} \frac{(n+k-l)^{2}}{\lambda_{r}+(n+k-l) \cdot \lambda_{f}}\right) \cdot \frac{\lambda_{r}+(n+i) \cdot \lambda_{f}}{(n+i)^{2}} \cdot \frac{n \cdot \mu}{(n+1+i) \cdot \lambda_{s}}\right\} \\
-\left\{\frac{n \cdot \mu}{(n+k+1) \cdot \lambda_{s}}+\left(\frac{\lambda_{f} \cdot \lambda_{u}}{\mu}\right)^{n+k} \cdot\left(\prod_{l=0}^{n+k-1} \frac{(n+k-l)^{2}}{\lambda_{r}+(n+k-l) \cdot \lambda_{f}}\right) \cdot \frac{\mu}{\lambda_{f}} \cdot\left(\frac{\lambda_{r}}{r \cdot \mu_{r}}+\frac{\lambda_{s}}{s \cdot \mu_{s}}\right)\right\}
\end{array}\right]
$$

We define the performable availability (PA) as the probability that the systems are in one of the availability states that meet a minimum performance threshold, such as the task waiting time. The time span difference in the order of magnitude between availability and waiting time analyses is very large. To overcome the difficulty, we distinguish the definition of $P A$ from the conventional definition of availability $(C A)$ as below equation (1).

$$
C A=\sum_{i=1}^{n-1}\left(P_{i, 0}+P_{U_{i, 0}}\right)+\sum_{i=0}^{k}\left(P_{n, i}+P_{U_{n, i}}\right)+\sum_{i=2}^{n+k} \sum_{j=1}^{r}\left(P_{R_{i, j}}+P_{R_{i, j}^{\prime}}\right)+\sum_{i=n}^{n+k-1} \sum_{j=1}^{s} P_{S_{i, j}}=1-\left(\sum_{i=2}^{n+k} P_{S_{i}^{\prime}}+P_{R_{1}}+P_{0}\right)
$$

Let $\Delta_{i}$ denote the indicator to determine that the system is available when $i$ primary components are functioning. Then, $\Delta_{i}=1$, if $d \leq D_{u}$ and $\Delta_{i}=0$, otherwise where $D_{u}$ denotes user-specified maximum waiting time, and $d$ the waiting time when the systems have $i$ primary components. We assume that the interarrival time of the transactions and the length of time required to process a transaction are independently and exponentially distributed random variables with rate $1 / \alpha, 1 / \beta$, respectively. The systems must have at least $m_{0}$ primary components functioning in order to meet the performability requirements with respect to task waiting time. And $\alpha / m_{0} \beta<1$ should be satisfied for the condition of ergodicity. Based on these assumptions, the system performability for PA can be solved by using an $\mathrm{M} / \mathrm{M} / \mathrm{m}_{0}$ queueing system. $W_{q}(t)$, the waiting time distribution of $\mathrm{M} / \mathrm{M} / \mathrm{m}_{0}$, is referred to as Erlang's $C$ formula, $C\left(m_{0}, \alpha / \beta\right)$ [14]. From this formula, the system is considered available if the condition of equation (1) is met. $W(d)=P\left[W_{q} \leq d\right] \geq \psi$ is obtained, where $\psi$ (psi) and $W_{q}$ represent acceptance ratio and a random variable which describes the amount of sojourn time in queues, respectively (equation (2)). From equations (1) and (2), performable availability $(P A)$ is defined as below:

$$
P A=\left\{\begin{array}{lr}
\sum_{i=1}^{n-1} \Delta_{i}\left(P_{i, 0}+P_{U_{i, 0}}\right)+\Delta_{n} \sum_{i=0}^{k}\left(P_{n, i}+P_{U_{n, i}}\right)+\Delta_{n-1}\left(\sum_{i=2}^{n+k} \sum_{j=1}^{r}\left(P_{R_{i, j}}+P_{R_{i, j}^{\prime}}\right)+\sum_{i=n}^{n+k-1} \sum_{j=1}^{s} P_{S_{i, j}}\right) & \text { if } n>m_{0} \\
\Delta_{n} P_{n, 0} & \text { if } n=m
\end{array}\right.
$$


Therefore, we merge the output of $\mathrm{M} / \mathrm{M} / \mathrm{m}_{0}$ queueing analysis with that of the steadystate probabilities of a ubiquitous computing system. At system initialization, the user sets the requirement of system availability ( $\Omega$,omega) and derives a utilization metric ( $\rho=\alpha / m_{0} \beta$ ) through periodic sampling of the average transaction arrival rate. Initially, the number of primary components satisfy the (user-defined) waiting time deadline $\left(D_{u}\right)$ through Erlang's $C$ formula (see equation (2)). Next, the decision is made over optimal redundancy levels by analyzing the performable availability with change of the number of primary or backup components (see equation (3)). The algorithm produces the optimal redundancy level of a ubiquitous computing system meeting the performability and availability as its output. When the systems need to handle heavy workload, the dynamic configuration algorithm should follow certain priority policy in its decision making process. For example, if the priority of static services is higher than that of dynamic services, more servers would be added to the systems for static service by using equation (4), where $S(t)$ is the number of servers for static service at time $t, A_{s}(t)$ the system availability for static service at time $t, L_{s}(t)$ the total workload in both systems, $M_{s}(t-1)$ : the maximum connection number of one server, and $\mathrm{S}(t-1)$ the number of servers for static service at time $t-1$.

$$
\text { if } L_{s}(t)>S(t) \cdot M_{s}(t-1) \text { or } A_{s}(t)<\Omega \text {, then } S(t)=S(t-1)+1
$$

\section{Performance Evaluation}

By using the system and workload parameters reported in Table 1, we can create simulation models based on well established traffic distributions. Similar to numerous observations on the network traffic, M. E. Crovell also characterized the web traffic as bursty[15]. As a result, we use heavy-tailed distributions to characterize the high variability and self-similar nature of the traffic patterns. The time to process a static request is proportional to the files size. The service time for a dynamic object is modeled according to hyper-exponential distribution. We performed experiments to find the optimal configuration for the ubiquitous computing systems using the following system-operating parameters $[8,9,10]$. The systems are analyzed at intervals of 1 year. Failure rate of a component is 2 times per year and repair time 12 hours. Rejuvenation schedule is 1 time per month and healthy systems may enter unstable states every 15 days. Switchover and rejuvenation time are 10 minutes and 20 seconds, respectively. As usual, the number of transactions varies with the time of the day. We assume that the average transaction rates are 4200 transactions/hour for

Table 1. Averages and standard deviations of the three daily periods

\begin{tabular}{|c|c|c|c|c|c|c|}
\hline \multirow{2}{*}{ Periods } & \multicolumn{2}{|c|}{ Mean number of requests } & \multicolumn{2}{|c|}{ Standard deviation } & \multirow{2}{*}{ Average } & $\begin{array}{c}\text { Standard } \\
\text { Deviation }\end{array}$ \\
\cline { 2 - 5 } & $2005 / 01 / 22$ & $2005 / 01 / 23$ & $2005 / 01 / 22$ & $2005 / 01 / 23$ & & 3.36 \\
\hline \hline $0: 00-8: 00$ & 31.50 & 26.75 & 19.71 & 19.40 & 29.13 & 34.39 \\
\hline $8: 00-16: 00$ & 135.75 & 137.75 & 42.52 & 136.75 & 1.41 \\
\hline $16: 00-24: 00$ & 93.37 & 82.00 & 15.88 & 16.23 & 87.63 & 7.95 \\
\hline
\end{tabular}


0AM-8AM, 5400 transactions/hour for 8AM-4PM., and 4800 transactions/hour for 4PM-0AM. Each component can process 1200 transaction per hour, and at least $90 \%$ of transactions have to meet 1-minute waiting time deadline. The systems must be available up to $99.99 \%$. To adequately approximate a deterministic sojourn time in rejuvenation and switchover states, the number of stages is set to 20 .

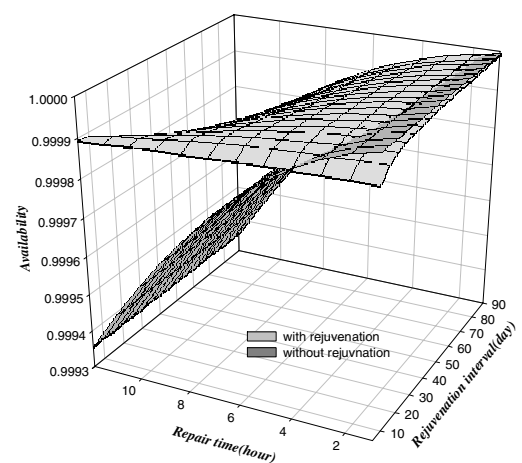

Fig. 4. System availability with respect to rejuvenation period and repair time

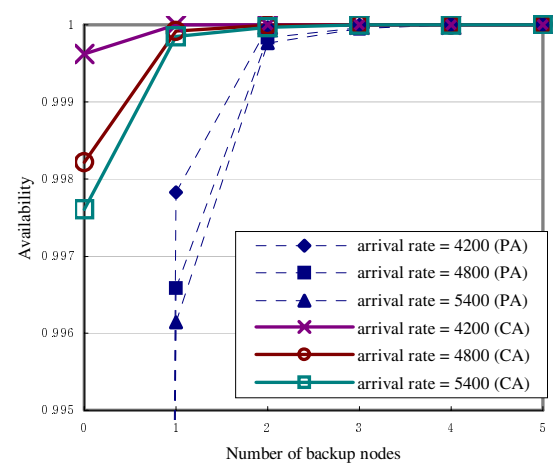

Fig. 6. Analysis according to transaction arrival rate

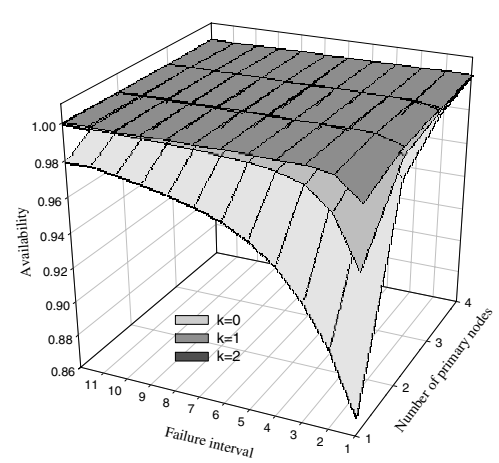

Fig. 5. Availability according to software rejuvenation process

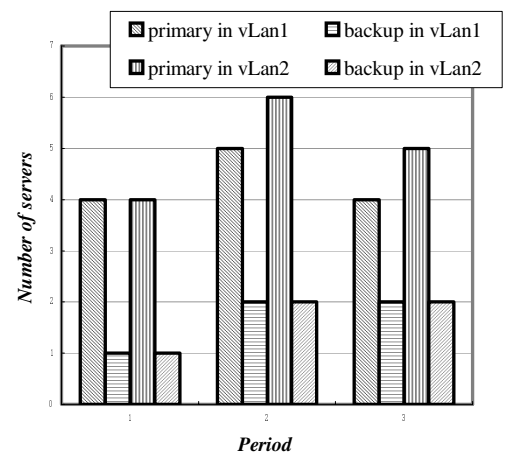

Fig. 7. The number of primary and backup server

The effect of software rejuvenation on availability of the systems is shown in Fig. 4, using the conventional availability defined in equation (1). As repair time becomes shorter, availability increases regardless of software rejuvenation, a result of software aging phenomenon, called heisenbugs. We note, however, systems with software rejuvenation can keep high availability because dormant software faults diminishes. In other words, software rejuvenation can reduce the frequency of failure in the ubiquitous computing systems. However, the result suggests that the system cannot meet its availability requirements of $99.99 \%$ if software rejuvenation period is too long. Fig. 5 shows the effects of failure rate and the number of backup 
components on the system availability. Spare system capacity is a necessity for software rejuvenation, implying that at the single system state $(1,0)$ software rejuvenation cannot improve availability. A duplex $(1,1)$ or multiplex $(v, w)$ system with a higher degree of redundancy is needed for availability improvement, where $v \geq$ 2 and $w \geq 2$. However, an excessively redundant system only provides marginal improvement on availability. For instance, the availability difference between a $(3,1)$ way system from that of a $(3,2),(4,1)$ or $(4,2)$-way system is very small. It is highly desirable to find a minimally configured system (of minimal cost) that can meet the system requirements. In Fig. 6, the $C A$ and $P A$ measures in three time slots are plotted with respect to transaction arrival rate, given that that the system has four primary components. The availability of a system with no backup components is too little to meet availability requirement (99.99\%). However, no further visible advantage can be gained if more than four backup components are added to the system. Fig. 7 describes how to optimize a system autonomously. Initially, the algorithm selects the $(7,0)$ to meet the waiting time deadline requirement. Next, alternatives to the initial choice, such as $(6,1),(5,2)$ and $(4,2)$ are examined in order to find the optimal point. After the search and computational processes, one can reduce to a $(3,2)$-way configuration from the $(7,0)$-way configuration without violating any requirements.

\section{Conclusion}

The ubiquitous computing systems that provide computing services to customers, dynamically need to meet not only the availability but also the performance requirements. In this paper, we develop a management mechanism for applying software rejuvenation, a proactive management method, into a ubiquitous computing system. It can automatically collect, aggregate, filter, track, analyze, and make plan to manage the ubiquitous computing system. Also, we validate the autonomic fault management scheme based on a workload model derived from the system log analysis with a scenario applicable to the run-time configuration phase. Moreover, this behavior such as human autonomous nervous system can be extended to other two phases of the application life cycle: the design phase and the deployment phase. In the future work, we will extend the proposed mechanism with an inference machine.

\section{References}

1. Satyanarayanan M.: Pervasive Computing: Vision and Challenges. IEEE Personal Communications (2001) 10-17.

2. Sun_Microsystems: J2EE Platform Specification (2002) http://java.sun.com/j2ee/

3. Microsoft: The Microsoft .NET Framework. Microsoft Press (2001)

4. Garg S., Moorsel A., Vaidyanathan K., Trivedi K.: A Methodology for Detection and Estimation of Software Aging. Proceedings of $9^{\text {th }}$ IEEE International Symposium on Software Reliability Engineering (1998) 282-292.

5. Sullivan M., Chillarehe R.: Software Defects and Their Impact on System Availability-A Study of Field Failures in Operating Systems. Proceedings of $21^{\text {st }}$ IEEE International Symposium on Fault-Tolerant Computing (1991) 2-9. 
6. Scott D.: Making Smart Investments to Reduce Unplanned Downtime. Tactical Guidelines Research Note TG-07-4033, Gartner Group (1999)

7. Huang Y., Kintala C., Kolettis N., Fultion N.: Software Rejuvenation: Analysis, Module and Applications. Proceedings of $25^{\text {th }}$ IEEE International Symposium on Fault-Tolerant Computing (1995) 318-390.

8. Trivedi K., Vaidyanathan K., Popstojanova K.: Modeling and Analysis of Software Aging and Rejuvenation. Proceedings of IEEE $33^{\text {rd }}$ Annual Simulation Symposium (2000) 270-279.

9. Park K., Kim S.: Availability Analysis and Improvement of Active/Standby Cluster Systems using Software Rejuvenation. The Journal of Systems Software, Vol. 61, No. 2. (2002) 121-128.

10. Choi C., Kim S.: Self-configuring Algorithm for Software Fault Tolerance in (n, k)-way Cluster Systems. Lecture Notes in Computer Science, Springer, Vol. 2667, No. 1. (2003) 742-751.

11. DMTF Inc.-Common Information Model. http://www.dmtf.org/standards/cim/

12. Lanfranchi G., et. al.: Toward a New Landscape of Systems Management in An autonomic Computing Environment. IBM System Journal, Vol. 42, No. 1. (2003) 119-128

13. J. Arlat, et. al.: Dependability of COTS Microkernel-based Systems. IEEE Transactions on Computers, Vol. 51, No. 2. (2002) 138-163.

14. Kanoun K., Borrel M.: Fault-tolerant Systems Dependability: Explicit Modeling of Hardware and Software Component-Interactions. IEEE Transactions on Reliability, Vol. 49, No. 4. (2000) 363-376.

15. Kleinrock, L.: Queueing Systems Volume I: Theory. Wiley (1975)

16. Crovell M. E. and Bestavros A.: Self-Similarity in World Wide Web Traffic: Evidence and Possible Causes, IEEE/ACM Transactions on Networking, Vol. 5, No. 6. (1997) 835-846. 